\title{
Avifauna in Logged-Over Forest of Upper Baleh, Sarawak
}

\author{
ANDREW ALEK TUEN*1, ATTIQQAH FADZILIAH SAPIAN ${ }^{1}$, KHATIJAH ISMAIL ${ }^{1}$, \\ CINDY PETER ${ }^{1}$, MOHD HASRI AL-HAFIZ HABA ${ }^{1} \&$ CH'IEN C LEE ${ }^{2}$ \\ ${ }^{1}$ Institute of Biodiversity and Environmental Conservation, Universiti Malaysia Sarawak, 94300 Kota \\ Samarahan, Sarawak, Malaysia; ${ }^{2}$ Peti Surat P. 60, Pejabat Pos Pending, 93457, Sarawak, Malaysia \\ *Corresponding author: aatuen@unimas.my \\ Received: 31 July 2018 \\ Accepted: 28 September 2018 \\ Published: 30 December 2018
}

\begin{abstract}
Commercial logging is a major economic activity in the Upper Baleh catchment, Sarawak, so logged-over forest is the dominant forest type there. Avifauna survey was conducted in the logged-over forest of Upper Baleh in November 2015 as part of the Upper Baleh Heart of Borneo Expedition. The objective of the survey was to collect baseline data on the avifauna species that inhabit the study area, their conservation status and feeding guilds. Both mist-net and observation method were used. A total of 95 species of birds was recorded: 36 species via mist-nets and 69 species via observation. Little spiderhunter was the dominant species, accounting for 33\% of mist-netted bird. Seven species are Totally Protected including six species of hornbills and a Great Argus Pheasant, while 18 other species are Protected under the Sarawak Wild Life Ordinance 1998. The majority of the birds are insectivorous (55.8\%), foraging either at ground level (babblers), along the tree trunks or branches (woodpeckers) or at the canopy (flycatchers). Omnivorous birds, which feed on two or more types of diet, accounted for $48.4 \%$ of the avifauna species recorded and these include bulbuls and hornbills. The diverse community of bird, including the protected species, makes the area an attractive birding destination for visitors since now part of the catchment has been gazetted as a national park. Avifauna's role as pollinating and dispersing agent will help the logged-over forest to recover.
\end{abstract}

Keywords: conservation status, feeding guilds, Heart of Borneo, logging roads

Copyright: This is an open access article distributed under the terms of the CC-BY-NC-SA (Creative Commons Attribution-NonCommercial-ShareAlike 4.0 International License) which permits unrestricted use, distribution, and reproduction in any medium, for non-commercial purposes, provided the original work of the author(s) is properly cited.

\section{INTRODUCTION}

The Baleh River is a tributary of Rajang and has its origin in the Nieuwenhuis highlands that forms the border between Sarawak and Central Kalimantan. It has a large catchment area of $12,433 \mathrm{~km}^{2}$ and contributes significantly to the Rajang River basin. For the Kenyah community of Long Singut who settled at Upper Baleh in the late 1960s, the river is the only way to go down to the main towns of Kapit and Sibu. Currently, the main economic activity affecting the forest and thus the habitats of wildlife in the Upper Baleh region is commercial logging. Shifting cultivation is generally confined to accessible areas next to the river and human settlement.

Commercial logging in Sarawak started soon after the Second World War (Aiken \& Leigh, 1992) focusing mainly on peatswamp species. Strong demand for tropical timber and favourable government policies had accelerated the growth of the timber industry to become the mainstay of the economy with log production peaking at $19 \mathrm{Mm}^{3}$ in 1990 (Hon \& Shibata, 2013). According to Bryan et al. (2013) about $364,489 \mathrm{~km}$ of logging roads were constructed in Sarawak, Sabah and Brunei between 1999 and 2009 alone, with Sarawak having the highest density of logging roads in Borneo (0.89 $\mathrm{km} / \mathrm{km}^{2}$ ). A major consequence of this logging activity in Sarawak is the reduction in intact forest, from $92,152 \mathrm{~km}^{2}$ in 1973 to $18,161 \mathrm{~km}^{2}$ in 2010 (Gaveau et al., 2014). These intact forests are likely to be logged or converted into other forms of designated land use since they are not legally protected.

In Sarawak, legal protection is given to 37 Totally Protected Areas (TPAs) equivalent to 483,682 hectares of land, which have been gazetted for biodiversity conservation and appreciation of nature (Sarawak Forest Department, 2017). These TPAs are the only land that can be considered safe from logging and cultivation. However, many TPAs do not have management presence and their boundaries are not clearly marked, so encroachment into these areas is a major concern (Gumal, 2007; Mohd Azlan \& Lawes, 2011). In addition, the majority of TPAs in Sarawak are less than $10,000 \mathrm{~km}^{2}$ in size and scattered throughout the State, with little regards for connectivity. This compromises their 
capacity to sustain viable population of large animals with large home range (Mohd Azlan \& Lawes, 2011).

Aware of the shortcomings of the current system of protected areas and conscious of the need to balance economic development and biodiversity conservation in Borneo, the government of Malaysia, Indonesia and Brunei agreed in February 2007 to commit funding and resources to promote sustainable development and conserve biodiversity under the Heart of Borneo (HoB) Initiative (http://wwf.panda.org). The Upper Baleh catchment is located in central Borneo and is part of the 22 million hectares under the HoB Initiative. The forest there is among the last to be logged circa 1990s, and also the least known in terms of its avifauna composition. Its avifauna has never been documented by earlier naturalists and explorers that put Borneo on the zoological map (Cranbrook \& Leh, 1983; Smythies, 1999; Tuen, 2005).

Hence a mini scientific expedition to Upper Baleh was jointly organized by Universiti Malaysia Sarawak (UNIMAS), Sarawak Forestry Department (SFD) and World Wide Fund for Nature (WWF) in November 2015. One of the components of this expedition was a survey of avifauna with the objective to produce a checklist of the bird species inhabiting the logged-over forest of Upper Baleh, Kapit Division, Sarawak, including their conservation status and feeding guilds. The results of the survey are presented in this paper.

\section{MATERIALS AND METHODS}

\section{Sampling Site}

Avifauna sampling was carried out using mistnets in the hill dipterocarp forest near Elite Honour Camp and by observation along the logging road heading east until the base of White Rock Mountain near Batu Tiban about $10 \mathrm{~km}$ from Indonesian Border. Elite Honour Camp (Figure 1) is one of the logging camps located on hill slope south of Baleh River, within a WTK logging concession area.

The mist-netting sites were located on a ridge about 300 meters above the sea level. The forest near the camp was relatively undisturbed because it was the water catchment for the camp while the 1000-meter site was logged about 10 years ago and comprised mainly of secondary growth forest. Active logging was being carried out in many parts of the concession area with the area near the base of White Rock Mountain being opened up for logging for the first time. The gaps created by falling timber trees and skid trails from earlier logging activity have reverted into secondary forests. Other secondary forest areas are fallow land, which is close to the river bank where it has been cleared for padi planting by the Long Singut Kenyah community.

\section{Sampling Method}

Mist-netting is a preferred method for bird survey when bird ringing is required or when more information is sought from each bird (Karr, 1981). Ten mist-nets were set up about 100 meters from Elite Honour Camp (Station 1, N01 ${ }^{\circ}$ 33' 32"; E114 11' 9) and another 10 nets were set up about 1000 meters east the camp (Station 2, N01'33' 35"; E114' 12' 7'). The mist-nets were operated between 6 am and $6 \mathrm{pm}$ from 2125 November 2015.

Observation was carried out opportunistically with the aid of binoculars (Nikon, 7x42) around the logging camp area and along the logging road from the camp to Long Singut in the east, then to the base of the White Rock Mountain where active logging is being carried out. The total distance of the logging road surveyed was $52 \mathrm{~km}$
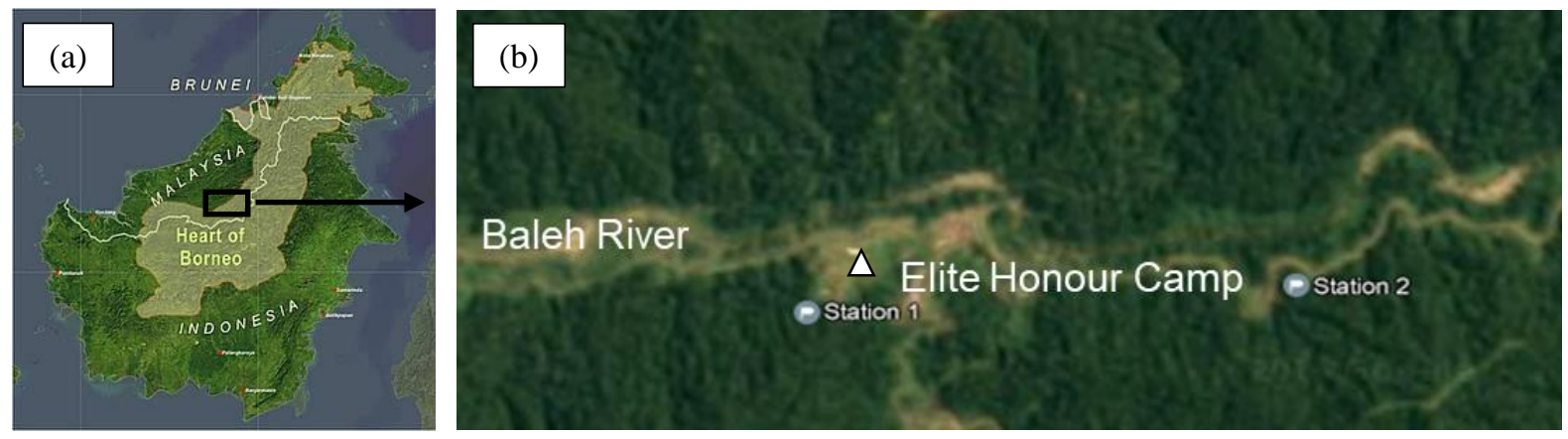

Figure 1. Map of Borneo showing location of Upper Baleh where the expedition was carried out (a) and avifauna sampling stations near Elite Honour Camp (b). $\triangle$ Location of Elite Honour Camp. 
and the elevation ranged from 220 to 760 meters above sea level. Birds were detected both via sighting and vocalisation; and species detected through vocalisation were recorded only if confirmed by sighting. Identification of feeding guilds followed Smythies (1999) and the naming of species followed Smythies (1999) and Phillipps (2014).

\section{RESULTS}

The combination of mist-net and observational method employed during this expedition yielded a total of 95 avifauna species (Table 1). Thirtysix species were recorded using mist-net while 69 were recorded using observational method. This list represents the first record of avifauna community for Upper Baleh catchment. Species such as Cattle Egret, Little Egret, Common Sandpiper, Grey Wagtail and Tree Sparrow (observed around the logging camp compound) are not strictly forest birds.

Of the 36 species caught using mist-net, the most abundant was Little Spiderhunter (54 individuals, 33\%). In contrast, 11 species were caught only once, including Crested Goshawk, Plaintive Cuckoo, Black and White Bulbul, Chestnut-naped Forktail, Malaysian Blue Flycatcher and Long-billed Spiderhunter. Although the cumulative graph of species caught using mist-net (Figure 2) appeared to have reached a plateau, more species would have been added to the list had more sites been sampled and sampling period prolonged.

Eighteen of the species that we recorded are listed as Protected under the Sarawak Wild Life Protection Ordinance 1998. These include eagles (4), woodpeckers (3), egrets (2), Common
Sandpiper, Rufous-backed Kingfisher, Imperial Pigeon, Garnet Pitta, Buffy Fish Owl, Hanging Parrot, White-rumped Shama, Chestnut-naped Forktail and Hill Myna. Seven of the species that we recorded are listed as Totally Protected under the same ordinance where these include six species of hornbills and the Great Argus Pheasant. All of the Totally Protected species were detected by observation only, which indicates the usefulness of this method for conservation assessment and thus, there is a need to train more students to be proficient in this method.

Three migrant species (Blue and White Flycatcher, Grey Wagtail and Cattle Egret) and three montane endemics species (Mountain Imperial Pigeon, Mountain Barbet and Little Cuckoo-Dove) were also identified during the observation. The egrets (Little Egret and Cattle Egret) were spotted flying up and down Baleh River. Only four of the species we recorded are endemic to Borneo, which are Mountain Barbet, Bornean Brown Barbet, Dusky Munia and Yellow-rumped Flowerpecker. None of the species recorded is categorized as Threatened according to IUCN Red List of Threatened Species 2018.

The majority of the birds are insectivorous $(55.8 \%)$, foraging either at ground level (babblers), along the tree trunks or branches (woodpeckers) or at the canopy (flycatchers). A further $18.9 \%$ of the birds also include insects as their secondary diet. Omnivorous birds, which feed on two or more types of diet accounted for $48.4 \%$ of the avifauna species recorded and these include bulbuls and hornbills.

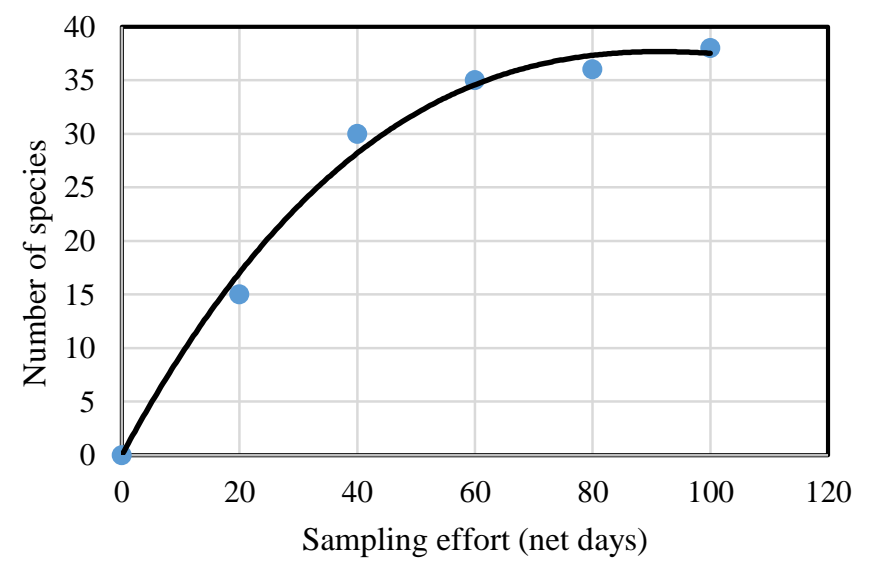

Figure 2. Cumulative graph of mist-netted birds recorded in Ulu Baleh in November 2015. 
Table 1. Summary of avifauna species recorded in Ulu Baleh in November 2015 (Status: WPO = Wild Life Protection Ordinance 1998, IUCN = IUCN Red List of Threatened Species 2015, P = Protected, TP $=$ Totally Protected, NA = not yet assessed, $\mathrm{LC}=$ Least Concern, NT $=$ Near Threatened, Guild represent the main feeding guild of the bird, $\mathrm{C}=$ carnivore, $\mathrm{F}=$ frugivore, $\mathrm{G}=$ granivore, $\mathrm{I}=$ insectivore, $\mathrm{N}$ = nectarivore, $\mathrm{O}=$ omnivore).

\begin{tabular}{|c|c|c|c|c|c|c|}
\hline \multirow[b]{2}{*}{ Family/Species } & \multicolumn{3}{|c|}{ Conservation Status } & \multicolumn{2}{|c|}{ Detection method } & \multirow[t]{2}{*}{ Guild } \\
\hline & WPO & IUCN & Residential & $\begin{array}{c}\text { Mist- } \\
\text { net }\end{array}$ & Observation & \\
\hline \multicolumn{7}{|l|}{ Accipitridae } \\
\hline Crested Goshawk (Accipiter trivigatus) & $\mathrm{P}$ & NA & Resident & 1 & & $\mathrm{C}$ \\
\hline Crested Serpent-eagle (Spilornis cheela) & $\mathrm{P}$ & $\mathrm{LC}$ & & & 2 & $\mathrm{C}$ \\
\hline Black Eagle (Ictinaetus malayensis) & $\mathrm{P}$ & LC & & & 1 & $\mathrm{C}$ \\
\hline Rufous-bellied Hawk-eagle (Spizaetus cirrhatus) & $\mathrm{P}$ & NA & & & 1 & $\mathrm{C}$ \\
\hline \multicolumn{7}{|l|}{ Ardeidae } \\
\hline Cattle Egret (Bubulcus ibis) & $\mathrm{P}$ & LC & Migrant & & 1 & $\mathrm{C}, \mathrm{I}$ \\
\hline Little Egret (Egretta garzetta) & $P$ & $\mathrm{LC}$ & $\begin{array}{l}\text { Migrant \& } \\
\text { Resident }\end{array}$ & & 4 & $\mathrm{C}, \mathrm{I}$ \\
\hline \multicolumn{7}{|l|}{ Scolopacidae } \\
\hline Common Sandpiper (Actitis hypoleucos) & $\mathrm{P}$ & $\mathrm{LC}$ & Resident & & 1 & $\mathrm{C}$ \\
\hline \multicolumn{7}{|l|}{ Phasianidae } \\
\hline Great Argus (Argusianus argus) & TP & NT & Resident & & 2 & I, F \\
\hline \multicolumn{7}{|l|}{ Columbidae } \\
\hline Mountain Imperial Pigeon (Ducula badia) & $\mathrm{P}$ & NA & $\begin{array}{l}\text { Montane } \\
\text { resident }\end{array}$ & & 3 & $\mathrm{~F}$ \\
\hline Emerald Dove (Chalcophaps indica) & - & & & 7 & & $\mathrm{~F}, \mathrm{G}$ \\
\hline Little Cuckoo-dove (Macropygia ruficeps) & & NA & $\begin{array}{l}\text { Submontane } \\
\text { resident }\end{array}$ & & 2 & $\mathrm{~F}, \mathrm{G}$ \\
\hline \multicolumn{7}{|l|}{ Psittacidae } \\
\hline Blue-crowned Hanging Parrot (Loriculus galgulus) & $\mathrm{P}$ & $\mathrm{LC}$ & Resident & & 2 & F, I \\
\hline \multicolumn{7}{|l|}{ Cuculidae } \\
\hline Violet Cuckoo (Chrysococcyx xanthorhynchus) & & $\mathrm{LC}$ & Resident & & 1 & $\mathrm{I}, \mathrm{F}$ \\
\hline Plaintive Cuckoo (Cacomantis merulinus) & - & LC & Resident & 1 & & $\mathrm{I}, \mathrm{F}$ \\
\hline Raffles's Malkoha (Phaenicophaeus chlorophaeus) & - & $\mathrm{LC}$ & Resident & & 4 & I \\
\hline $\begin{array}{l}\text { Chestnut-breasted Malkoha } \\
\text { (Phaenicophaeus curvirostris) }\end{array}$ & & & Resident & & 1 & I \\
\hline Lesser Coucal (Centropus bengalensis) & - & $\mathrm{LC}$ & Resident & & 1 & $\mathrm{I}, \mathrm{O}$ \\
\hline \multicolumn{7}{|l|}{ Strigidae } \\
\hline Buffy Fish-owl (Ketupa ketupu) & $P$ & $\mathrm{LC}$ & Resident & & 1 & $\mathrm{C}$, \\
\hline \multicolumn{7}{|l|}{ Apodidae } \\
\hline Silver-rumped Needletail (Rhaphidura leucopygialis) & & & Resident & & 4 & I \\
\hline \multicolumn{7}{|l|}{ Trogonidae } \\
\hline Red-naped Trogon (Harpactes kasumba) & & $\mathrm{LC}$ & Resident & & 1 & I \\
\hline \multicolumn{7}{|l|}{ Alcedinidae } \\
\hline Rufous-backed Kingfisher (Ceyx rufidorsus) & $\mathrm{P}$ & $\mathrm{LC}$ & Resident & 4 & 1 & I \\
\hline \multicolumn{7}{|l|}{ Meropidae } \\
\hline Red-bearded Bee-Eater (Nyctyornis amictus) & - & & Resident & & 1 & I \\
\hline \multicolumn{7}{|l|}{ Bucerotidae } \\
\hline White-crowned Hornbill (Berenicornis comatus) & TP & LC & Resident & & 1 & $\mathrm{~F}, \mathrm{O}$ \\
\hline Bushy-crested Hornbill (Anorrhincus galeritus) & TP & LC & Resident & & 9 & $\mathrm{~F}, \mathrm{O}$ \\
\hline Black Hornbill (Anthracoceros malayanus) & TP & NT & Resident & & 2 & $\mathrm{~F}, \mathrm{O}$ \\
\hline Oriental Pied Hornbill (Anthrococeros albirostis) & TP & LC & Resident & & 2 & $\mathrm{~F}, \mathrm{O}$ \\
\hline Rhinoceros Hornbill (Buceros rhinoceros) & TP & NT & Resident & & 1 & $\mathrm{~F}, \mathrm{O}$ \\
\hline Wreathed Hornbill (Rhyticeros undulatus) & $\mathrm{TP}$ & & Resident & & 2 & $\mathrm{~F}, \mathrm{O}$ \\
\hline \multicolumn{7}{|l|}{ Megalaimidae } \\
\hline Bornean Brown Barbet (Calorhamphus fuliginosus) & - & $\mathrm{LC}$ & Endemic & & 3 & I, F \\
\hline Red-crowned Barbet (Megalaima rafflesii) & - & NT & Resident & & 1 & $\mathrm{~F}$ \\
\hline Red-throated Barbet (Megalaima mystacophanos) & - & NT & Resident & & 2 & $\mathrm{~F}, \mathrm{O}$ \\
\hline Yellow-crowned Barbet (Megalaima henricii) & - & NT & $\begin{array}{l}\text { Resident, sub- } \\
\text { montane }\end{array}$ & & 3 & $\mathrm{~F}, \mathrm{O}$ \\
\hline Blue-eared Barbet (Megalaima australis) & - & $\mathrm{LC}$ & Resident & & 2 & $\mathrm{~F}$ \\
\hline Gold-whiskered Barbet (Megalaima chrysopogon) & - & $\mathrm{LC}$ & Resident & & 1 & $\mathrm{~F}, \mathrm{O}$ \\
\hline Mountain Barbet (Megalaima monticola) & - & $\mathrm{LC}$ & $\begin{array}{l}\text { Montane } \\
\text { endemic }\end{array}$ & & 1 & $\mathrm{~F}, \mathrm{I}$ \\
\hline \multicolumn{7}{|l|}{ Picidae } \\
\hline Rufous Piculet (Sasia abnormis) & $\mathrm{P}$ & $\mathrm{LC}$ & Resident & 3 & & I \\
\hline
\end{tabular}


Table 1. Continued.

\begin{tabular}{|c|c|c|c|c|c|c|}
\hline \multirow[b]{2}{*}{ Family/Species } & \multicolumn{3}{|c|}{ Conservation Status } & \multicolumn{2}{|c|}{ Detection method } & \multirow[t]{2}{*}{ Guild } \\
\hline & WPO & IUCN & Residential & $\begin{array}{c}\text { Mist- } \\
\text { net }\end{array}$ & Observation & \\
\hline \multicolumn{7}{|l|}{ Picidae } \\
\hline Crimson-winged Woodpecker (Picus puniceus) & $\mathrm{P}$ & LC & Resident & & 1 & I \\
\hline Maroon Woodpecker (Blythipicus rubiginosus) & $\mathrm{P}$ & $\mathrm{LC}$ & Resident & 3 & & I \\
\hline \multicolumn{7}{|l|}{ Pittidae } \\
\hline Garnet Pitta (Pitta granatina) & $\mathrm{P}$ & $\mathrm{LC}$ & Resident & & 1 & I \\
\hline \multicolumn{7}{|l|}{ Campephagidae } \\
\hline Black-winged Flycatcher-shrike (Hemipus hirundinaceus) & - & LC & Resident & & 2 & I \\
\hline Bar-winged Flycatcher-shrike (Hemipus picatus) & - & $\mathrm{LC}$ & $\begin{array}{l}\text { Montane } \\
\text { Resident }\end{array}$ & & 1 & I \\
\hline \multicolumn{7}{|l|}{ Eurylaimidae } \\
\hline Green Broadbill (Calyptomena viridis) & - & NT & Resident & 2 & & $\mathrm{~F}$ \\
\hline Black-and-yellow Broadbill (Eurylaimus ochromalus) & - & NT & Resident & & 1 & I \\
\hline Banded Broadbill (Eurylaimus javanicus) & - & LC & Resident & & 1 & I \\
\hline \multicolumn{7}{|l|}{ Hirundinidae } \\
\hline Pacific Swallow (Hirundo tahitica) & - & $\mathrm{LC}$ & Resident & & 23 & I \\
\hline \multicolumn{7}{|l|}{ Motacillidae } \\
\hline Grey Wagtail (Motacilla cinerea) & - & $\mathrm{LC}$ & Migrant & & 1 & I \\
\hline \multicolumn{7}{|l|}{ Pycnonotidae } \\
\hline Buff-vented Bulbul (Iole olivacea) & - & & Resident & 3 & 2 & F, I \\
\hline Finsch's Bulbul (Criniger finschii) & - & & $\begin{array}{l}\text { Submontane } \\
\text { Resident }\end{array}$ & & 2 & $\mathrm{I}, \mathrm{F}$ \\
\hline Hairy-backed Bulbul (Tricholestes criniger) & - & & Resident & 6 & & $\mathrm{I}, \mathrm{F}$ \\
\hline Black-headed Bulbul (Pycnonotus atriceps) & - & LC & Resident & & 2 & $\mathrm{~F}, \mathrm{I}$ \\
\hline Olive-winged Bulbul (Pycnonotus plumosus) & - & LC & Resident & & 1 & $\mathrm{I}, \mathrm{F}$ \\
\hline Red-eyed Bulbul (Pycnonotus brunneus) & - & LC & Resident & 2 & & $\mathrm{I}, \mathrm{F}$ \\
\hline Scaly-breasted Bulbul (Pycnonotus squamatus) & - & & $\begin{array}{l}\text { Submontane } \\
\text { Resident }\end{array}$ & & 2 & $\mathrm{~F}, \mathrm{I}$ \\
\hline Spectacled Bulbul (Pycnonotus erythropthalmos) & - & LC & Resident & 8 & & $\mathrm{I}, \mathrm{F}$ \\
\hline Grey-bellied Bulbul (Pycnonotus cyaniventris) & - & & Resident & & 1 & $\mathrm{~F}, \mathrm{I}$ \\
\hline Grey-cheeked Bulbul (Alophoixus bres) & - & LC & Resident & 3 & & $\mathrm{I}, \mathrm{F}$ \\
\hline Yellow-bellied Bulbul (Alophoixus phaeocephalus) & - & LC & Resident & 8 & & $\mathrm{I}, \mathrm{F}$ \\
\hline Black and White Bulbul (Pycnonotus melanoleucos) & - & LC & Resident & 1 & & F, I \\
\hline \multicolumn{7}{|l|}{ Turdidae } \\
\hline Oriental Magpie-Robin (Copyschus saularis) & - & LC & Resident & & 1 & I \\
\hline White-rumped Shama (Copyschus malabaricus) & $\mathrm{P}$ & LC & Resident & 4 & 2 & I \\
\hline Chestnut-naped Forktail (Enicurus ruficapillus) & $\mathrm{P}$ & LC & Resident & 1 & & I \\
\hline \multicolumn{7}{|l|}{ Timaliidae } \\
\hline Black-capped Babbler (Pellorneum capistratum) & - & $\mathrm{LC}$ & Resident & & 2 & I \\
\hline Ferruginous Babbler (Trichastoma bicolor) & - & $\mathrm{LC}$ & Resident & 2 & 1 & I \\
\hline Short-tailed Babbler (Malacocincla malaccense) & - & NT & Resident & 3 & 1 & I \\
\hline Scaly-crowned Babbler (Malacopteron cinereum) & - & LC & Resident & 1 & & I \\
\hline Sooty-capped Babbler (Malacopteron affine) & - & LC & Resident & 1 & & I \\
\hline Rufous-crowned Babbler (Malacopteron magnum) & - & LC & Resident & 2 & & I \\
\hline Striped Tit Babbler (Macronous gularis) & - & LC & Resident & & 3 & I \\
\hline Fluffy-backed Tit Babbler (Macronous ptilosus) & - & NT & Resident & 4 & 2 & I \\
\hline Black-throated Babbler (Stachyris nigricollis) & - & NT & Resident & 3 & & I \\
\hline Chestnut-winged Babbler (Stachyris erythroptera) & - & LC & Resident & 2 & 2 & I \\
\hline Chestnut-rumped Babbler (Stachyris maculata) & - & LC & Resident & 5 & & I \\
\hline Rufous-fronted Babbler (Stachyris rufifrons) & - & LC & Resident & & 1 & I \\
\hline Grey-headed Babbler (Stachyris poliocephala) & - & LC & Resident & 7 & & I \\
\hline \multicolumn{7}{|l|}{ Eupetidae } \\
\hline Brown Fulvetta (Alcippe brunneicauda) & - & NA & Resident & & 2 & $\mathrm{I}, \mathrm{F}$ \\
\hline \multicolumn{7}{|l|}{ Pardalotidae } \\
\hline Rufous-tailed Tailorbird (Orthotomus sericeus) & - & LC & Resident & 1 & 1 & I \\
\hline \multicolumn{7}{|l|}{ Muscicapidae } \\
\hline Pied Fantail (Rhipidura javanica) & - & $\mathrm{LC}$ & Resident & & 2 & I \\
\hline Blue-and-white Flycatcher (Cyanoptila cyanomelana) & - & LC & Migrant & & & $\mathrm{I}, \mathrm{F}$ \\
\hline Malaysian Blue Flycatcher (Cyornis turcosus) & - & LC & Resident & 1 & 1 & I \\
\hline Rufous-winged Philentoma (Philentoma pyrhoptera) & - & LC & Resident & 1 & & I \\
\hline
\end{tabular}


Table 1. Continued.

\begin{tabular}{|c|c|c|c|c|c|c|}
\hline \multirow[b]{2}{*}{ Family/Species } & \multicolumn{3}{|c|}{ Conservation Status } & \multicolumn{2}{|c|}{ Detection method } & \multirow[t]{2}{*}{ Guild } \\
\hline & WPO & IUCN & Residential & $\begin{array}{c}\text { Mist- } \\
\text { net }\end{array}$ & Observation & \\
\hline \multicolumn{7}{|l|}{ Muscicapidae } \\
\hline Grey-headed Canary Flycatcher (Culicicapa ceylonensis) & & LC & Resident & & 1 & I \\
\hline \multicolumn{7}{|l|}{ Dicaeidae } \\
\hline Yellow-breasted Flowerpecker (Prionochilus maculates) & - & $\mathrm{LC}$ & Resident & 4 & & $\mathrm{I}, \mathrm{F}$ \\
\hline $\begin{array}{l}\text { Yellow-rumped Flowerpecker } \\
\text { (Prionochilus xyanthopygius) }\end{array}$ & - & & Endemic & 8 & & $\mathrm{~N}, \mathrm{I}, \mathrm{F}$ \\
\hline \multicolumn{7}{|l|}{ Nectariniidae } \\
\hline Purple-naped Sunbird (Hyprogramma hypogrammicum) & - & $\mathrm{LC}$ & Resident & 4 & & $\mathrm{~N}, \mathrm{I}, \mathrm{F}$ \\
\hline Plain Sunbird (Anthreptes simplex) & - & LC & Resident & 1 & 1 & $\mathrm{~N}, \mathrm{I}, \mathrm{F}$ \\
\hline Temminck's Sunbird (Aethopyga temminckii) & - & $\mathrm{LC}$ & Resident & & 3 & $\mathrm{~N}, \mathrm{I}$ \\
\hline Little Spiderhunter (Arachnothera longirostra) & - & LC & Resident & 54 & & $\mathrm{~N}, \mathrm{I}$ \\
\hline Long-billed Spiderhunter (Arachnothera robusta) & - & LC & Resident & 1 & & $\mathrm{~N}, \mathrm{I}$ \\
\hline Thick-billed Spiderhunter (Arachnothera crassirostris) & - & $\mathrm{LC}$ & Resident & & 1 & $\mathrm{~N}, \mathrm{I}$ \\
\hline \multicolumn{7}{|l|}{ Sturnidae } \\
\hline Hill Myna (Gracula religiosa) & $\mathrm{P}$ & $\mathrm{LC}$ & Resident & & 2 & $\mathrm{~F}, \mathrm{O}$ \\
\hline \multicolumn{7}{|l|}{ Passeridae } \\
\hline Eurasian Tree Sparrow (Passer montanus) & - & $\mathrm{LC}$ & Resident & & 5 & $\mathrm{G}$ \\
\hline \multicolumn{7}{|l|}{ Estrildidae } \\
\hline Dusky Munia (Lonchura fuscans) & - & $\mathrm{LC}$ & Endemic & & 4 & G, I, F \\
\hline Black-headed Munia (Lonchura malacca) & - & $\mathrm{LC}$ & Resident & & 7 & G \\
\hline \multicolumn{7}{|l|}{ Irenidae } \\
\hline Asian Fairy Bluebird (Irena puella) & - & LC & Resident & 2 & 2 & $\mathrm{~F}, \mathrm{I}$ \\
\hline \multicolumn{7}{|l|}{ Corvidae } \\
\hline Slender-billed Crow (Corvus enca) & - & $\mathrm{LC}$ & Resident & & 8 & $\mathrm{I}, \mathrm{F}$ \\
\hline
\end{tabular}

\section{DISCUSSION}

The main limitation of this study is that the survey covers a very small area $(52-\mathrm{km}$ of logging roads) and only two mist-netting sites over a very short time (five days). However we have tried to increase the efficiency of our survey by combining both observational and mistnetting method leading to a haul of 95 species: 69 detected by observation and 36 using mistnet. Whitman et al. (1997) also reported more species recorded using observational method compared to mist-nets. Our data is also probably biased towards birds that are still common and easily detected due to their active and noisy behaviour. The bird list reported here represents the first record for Upper Baleh where this could provide a snapshot of what tourists expect to see if they come to this area as well as a reference for future work in the area.

Logging and shifting cultivation was still going on in the Upper Baleh area during the time of the expedition. Selective logging such as the one practiced in Upper Baleh catchment can remove about $54 \%$ of trees greater than $30 \mathrm{~cm}$ diameter at breast height (dbh) and a further $13 \%$ are damaged incidentally due to falling timber trees, construction of skid trails and logging roads (Bennett \& Gumal, 2001) while Berry et al. (2010) reported $21 \%$ of trees were removed by direct logging and another $32 \%$ by collateral damage associated with logging operation. Selective logging affects all bird species within the forest through opening of the canopy, intrusion of sunlight, drying, temperature increase, soil erosion, reduction of leaf litter, and effects on the invertebrates, flower and fruit production and changes in the predator community. The effect would be more devastating especially if the timber trees and other affected plants are food sources, nesting sites or place of refuge for Threatened or Protected bird species (Johns, 1988).

Lambert (1992) found that the abundance of some species of birds increased in selectively logged forests in Peninsular Malaysia compared to unlogged forests, while the abundance of other species decreased. An observational study conducted in Seram Island, Indonesia found that the avifauna diversity was lower in logged compared to unlogged forest (Marsden 1998). Repeatedly logged forest retained about $75 \%$ bird species richness of unlogged forest (Edwards et al., 2011).

The effect of logging on avifauna communities seems to depend on species traits such as size of birds and their feeding heights and guilds, and 
logging practices (Cleary et al., 2007; Burivalova et al., 2015; Hamer et al., 2015). Large avifauna species seemed to be particularly vulnerable because of their greater metabolic needs and habitat range which are disrupted by logging activities (Constantini et al., 2016). Logging appeared to have the strongest negative effect on the abundance of hornbills (Cleary et al., 2007; Naniwadekar et al., 2015) perhaps because of selective removal of large trees which are more likely to have tree holes that are used by birds for nesting. This can have negative implications on forest regeneration since hornbills are one of the most effective agents of seed dispersal (Kitamura, 2011). Six species of hornbills (out of eight species present in Borneo) were recorded in the Upper Baleh study area, suggesting either the logging intensity practiced by the company is not as severe as in other areas or the effects of logging are not complete since logging operation is still on-going. Insectivorous birds are particularly vulnerable to habitat disturbance (Sekercioğlu et al., 2002, Gray et al., 2007), perhaps because they constitute the majority of feeding guilds. However, this seem to depend on their trophic level, as well as their body size and foraging heights. Studies with babblers showed that large ground-feeding species occupying high trophic positions were more adversely affected than small understory-feeders with lower trophic positions (Hamer et al., 2015). This is attributed to a change in leaf litter arthropod composition (Burghouts et al., 1992), which in turn affect the abundance of their predators. In Upper Baleh, $53 / 95$ avifauna species $(55.7 \%)$ are insectivorous and a further 18 species that are categorized as omnivorous have insects in their diet. The predominance of insectivores in primary forest and agroforest was also reported recently by Attiqqah et al. (2017).

Logging roads can have a devastating impact on ecologically specialized species that cannot adapt to disturbances in their ecosystem (Laurance et al., 2009, Edwards et al., 2017). Logging roads open up the previously inaccessible areas to hunters making game animals especially more vulnerable. For example, an estimated $29,000 \mathrm{~kg}$ of wild meat was consumed by a population of 167 workers, mainly Iban, at Nanga Gaat logging camp located within the Baleh catchment, of which bearded pig constitute $71.4 \%$ (Dahaban et al., 1996). Although birds are not normally the target, sometimes they are trapped for food as well as for pet, especially Blue-crowned Hanging Parrots. A few parrots were kept as pets in cages by the Long Singut community within the Upper Baleh study area. In addition, the logging roads and skid trails do not only remove trees but also disturb the soil where these may have either positive impacts (exposes ground dwelling organisms e.g. worms, upon which bird feeds on) or negative impacts (destroys food source, nests and refuge for ground dwelling birds).

Besides logging, there was also conversion of forests into rice fields by the villagers of Long Singut. The process of land clearing for rice cultivation by the community is devastating for wildlife because it involves not only felling of trees but burning of the dried vegetation before planting can take place. The area affected at any one time is small, generally not more than one hectare per family, and after harvesting the land is usually left to grow back into secondary forest. Other threat includes the carnivorous pets (cats and dogs) owned by the logging community as well as the local community of Long Singut; these pets may harass wildlife, including birds.

Avifauna plays an important role in maintaining ecological balance through the services they provided, some of which can directly or indirectly benefit humans. These services include flower pollination and seed dispersers and the birds are therefore serve as important agents in the forest regeneration and recovery from disturbance. Other birds such as owls, hawks and eagles that feed on small vertebrates and the insectivorous birds such as babblers play an important role in biological pest control.

The ecosystem and biodiversity of Upper Baleh may be spared from further damage with the implementation of the HoB programs and initiatives, which may include gazetting the study area as a national park. The firm stance of Sarawak State government against illegal logging (New Straits Times, 2014), including by not issuing new timber license, will eventually allow the ecosystem to recover. Both the HoB Initiative and hydroelectric power dam further downstream has put the area under greater scrutiny from conservation-minded organization. This scrutiny and the greater awareness due to publicity and findings of the expedition has prompted the Sarawak Government to recently announce the gazettement of 66,721 ha of Upper Baleh as a national park (Sarawak Forest Department, 2018). The small Kenyah community in Long 
Singut are not expected to cause further damage to the ecosystem if they are given alternative means of livelihood under the HoB Initiative or better employment opportunities with the power supply company.

\section{CONCLUSION}

A total of 95 species were recorded during the expedition, including four endemic, seven Totally Protected and 18 Protected species. Insectivores and omnivores are the major feeding guilds of these birds. This diverse community of bird, including the presence of protected and colourful species, makes the area an attractive birding destination. Avifauna's role as pollinating and dispersing agent will help the logged and cultivated forest to recover.

\section{ACKNOWLEDGEMENTS}

The authors would like to thank Universiti Malaysia Sarawak, Forest Department Sarawak, WWF-Malaysia and Sarawak Energy Berhad for organizing and supporting the expedition, WTK Holdings for logistic support in the field and the Long Singut Kenyah community for field assistance.

\section{REFERENCES}

Aiken, S.R. \& Leigh, C.H. (1992). Vanishing rain forests: the ecological transition in Malaysia. Oxford, UK: Clarendon Press.

Attiqqah, F.S., Mohd-Azlan, J., Khatijah, I., SingTyan, P., Rahah, M.Y., Mohd Hasri Al-Hafiz, H., Isa, S. \& Tuen, A.A. (2017). Diversity and functional guild of understorey birds in different types of forest in Ulu Baram, Sarawak. Paper presented at UNIMAS Silver Jubilee Conference 2017, Pullman Hotel, Kuching.

Bennett, E.L. \& Gumal, M.T. (2001). The interrelationships of commercial logging, hunting and wildlife in Sarawak. The cutting edge, conserving wildlife in logged forests. New York, USA: Columbia University Press. Pp 359-374.

Berry, N.J., Phillips, O.L., Lewis, S.L., Hill, J.K., Edwards, D.P., Tawatao, N.B. \& Ong, R.C. (2010). The high value of logged tropical forests: lessons from northern Borneo. Biodiversity and Conservation, 19(4): 985-997.

Bryan, J.E., Shearman, P.L., Asner, G.P., Knapp, D.E., Aoro, G. \& Lokes, B. (2013). Extreme differences in forest degradation in Borneo: comparing practices in Sarawak, Sabah, and Brunei. PLoS ONE 8(7): e69679.
Burghouts, T., Ernsting, G., Korthals, G. \& De Vries, T. (1992). Litterfall, leaf litter decomposition and litter invertebrates in primary and selectively logged dipterocarp forest in Sabah, Malaysia. Philosophical Transactions of the Royal Society London B, 335(1275): 407-416.

Burivalova, Z., Lee, T.M., Giam, X., Şekercioğlu, Ç.H., Wilcove, D.S. \& Koh, L.P. (2015). Avian responses to selective logging shaped by species traits and logging practices. Proceedings of the Royal Society B, 282: 20150164.

Cleary, D., Boyle, T., Setyawati, T., Anggraeni, C., Van Loon, E. \& Menken, S. (2007). Bird species and traits associated with logged and unlogged forest in Borneo. Ecological Applications, 17(4): 1184-1197.

Cranbrook, Earl of. \& Leh, C.M.U. (1983). A history of zoology in Sarawak. Sarawak Museum Journal XXXII, 53: 15-33.

Constantini, D., Edwards, D.P. \& Simons, M.J.P. (2016). Life after logging in tropical forests of Borneo: a meta-analysis. Biological Conservation, 196: $182-188$.

Dahaban, Z., Nordin, M. \& Bennett, E.L. (1996). Immediate effects on wildlife of selective logging in a hill dipterocarp forest in Sarawak: mammals. In Edwards, D.S., Booth, W.E. \& Choy, S.C. (Eds.). Tropical Rainforest Research - Current Issues. Monographiae Biologicae, Vol 74. Springer, Dordrecht.

Edwards, D.P., Larsen, T.H., Docherty, T.D., Ansell, F.A., Hsu, W.W., Derhé, M.A. \& Wilcove, D.S. (2011). Degraded lands worth protecting: the biological importance of Southeast Asia's repeatedly logged forests. Proceedings of the Royal Society of London B: 278(1702): 82-90.

Edwards, F.A., Finan, J., Graham, L.K., Larsen, T.H., Wilcove, D.S., Hsu, W.W., Chey, V.K. \& Hamer, K.C. (2017). The impact of logging roads on dung beetle assemblages in a tropical rainforest reserve. Biological Conservation, 205: 85-92.

Gray, M.A., Baldauf, S.L., Mayhew, P.J. \& Hill, J.K. (2007). The response of avian feeding guilds to tropical forest disturbance. Conservation Biology, 21:133-141.

Gaveau, D.L.A., Sloan, S., Molidena, E., Yaen, H., Sheil, D., Abram, N.K., Ancrenac, M., Nasi, R., Quinones, M., Wielaard, N. \& Meijaard, E. (2014). Four decades of forest persistence, clearance and logging on Borneo. PLOS ONE, 9(7): e101654. 
Gumal, M. (2007). Keynote address: TPA management and communities: Conserving totally protected areas with rural communities living in and around these areas. Proceedings of the 8th Hornbill Workshop on Protected Areas and Biodiversity Conservation. Pp 226-236.

Hamer, K.C., Newton, R.J., Edwards, F.A., Benedick, S., Bottrell, S.H. \& Edwards, D.P. (2015). Impacts of selective logging on insectivorous birds in Borneo: the importance of trophic position, body size and foraging height. Biological Conservation, 188: 82-88.

Hon, J. \& Shibata, S. (2013). A review on land use in the Malaysian State of Sarawak, Borneo and recommendations for wildlife conservation inside production forest environment. Borneo Journal of Resource Science and Technology, 3(2): 22-35.

Johns, A. (1988). Effects of "selective" timber extraction on rain forest structure and composition and some consequences for frugivores and folivores. Biotropica, 20(1): 3137.

Karr, J.R. (1981). Surveying birds with mist nets. Studies in Avian Biology, 6: 62-67.

Kitamura, S. (2011). Frugivory and seed dispersal by hornbills (Bucerotidae) in tropical forest. Acta Oecologia, 37(6): 531-541.

Lambert, F.R. (1992). The consequences of selective logging for Bornean lowland forest birds. Philosophical Transactions of the Royal Society London B, 335(1275): 443-457.

Laurance, W.F., Goosem, M. \& Laurance, S.G. (2009). Impacts of roads and linear clearings on tropical forests. Trends in Ecology \& Evolution, 24(12), 659-669.

Marsden, S.J. (1998). Changes in bird abundance following selective logging on Seram, Indonesia. Conservation Biology, 12(3): 605-611.
Mohd Azlan, J. \& Lawes, M.J. (2011). The efficacy of protected areas and future challenges for wildlife conservation in Sarawak. In Mokhtar, M. \& Abdul Halim, J.C. (Eds.), RIMBA2: Regional Sustainable Development in Malaysia and Australia. Bangi, Malaysia: Lestari. Pp 136-146.

Naniwadekar, R., Shukla, U., Isvaran, K. \& Datta, A. (2015). Reduced hornbill abundance associated with low seed arrival and altered recruitment in a hunted and logged tropical forest. PLoS ONE, 10(3), e0120062.

New Straits Times (20 November 2014). Tougher laws needed to fight illegal logging. https://www.nst.com.my/news/2015/09. Downloaded on 31 July 2018.

Phillipps, Q. (2014). Phillipps' Field Guide to the Birds of Borneo. Oxford: John Beaufoy Publishing.

Sarawak Forest Department (2018) http://www.forestry.sarawak.gov.my/page-0-2571012-National-Parks.html. Downloaded on 31 July 2018.

Şekercioḡlu, Ç.H., Ehrlich, P.R., Daily, G.C., Aygen, D., Goehring, D. \& Sandí, R.F. (2002). Disappearance of insectivorous birds from tropical forest fragments. Proceedings of the National Academy of Sciences, 99(1): 263-267.

Smythies, B.E. (1999). Birds of Borneo. $4^{\text {th }}$ edition. Kota Kinabalu, Malaysia: Natural History Publications.

Tuen, A.A. (2005). A fauna study of the Rajang River Basin. Proceedings of the $7^{\text {th }}$ Hornbill Conference, Kuching, 2005. Pp 115-134.

Whitman, A.A., Hagan III, J.M. \& Brokaw, N.V. (1997). A comparison of two bird survey techniques used in a subtropical forest. Condor, 99: 955-965. 